\title{
Allergen immunotherapy: the growing role of observational and randomised trial "real-world evidence"
}

\author{
Giovanni Paoletti ${ }^{1}$, Danilo Di Bona ${ }^{2}$, Derek $\mathrm{Chu}^{3}$, Davide Firinu ${ }^{4}$, Enrico Heffler ${ }^{1}$, Ioana \\ Agache $^{5}$, Marek Jutel ${ }^{6}$, Ludger Klimek ${ }^{7}$, Oliver Pfaar ${ }^{8}$, Ralph Mosges ${ }^{9}$, Audrey \\ DunnGalvin $^{10}$, Jon Genuneit ${ }^{11}$, Hans Jürgen Hoffmann ${ }^{12}$, and Giorgio Walter Canonica ${ }^{13}$ \\ ${ }^{1}$ IRCCS Istituto Clinico Humanitas \\ ${ }^{2}$ Università degli Studi di Bari-Aldo Moro \\ ${ }^{3}$ McMaster University \\ ${ }^{4}$ University of Cagliari \\ ${ }^{5}$ Transylvania University of Brasov \\ ${ }^{6}$ Wroclaw Medical University \\ ${ }^{7}$ Zentrum für Rhinologie und Allergologie \\ ${ }^{8}$ University Hospital Marburg, Philipps-Universität Marburg, Marburg, Germany. \\ ${ }^{9}$ Universitat $\mathrm{zu}$ Koln \\ ${ }^{10}$ University College Cork (UCC) \\ ${ }^{11}$ Ulm University \\ ${ }^{12}$ Aarhus University Hospital \\ ${ }^{13}$ IRCCS Humanitas Research Hospital
}

January 15, 2021

\begin{abstract}
Although there is a considerable body of knowledge about allergen immunotherapy (AIT), there is a lack of data on the reliability of real-world evidence (RWE) in AIT and consequently, a lack of information on how AIT effectively works in real life. To address the current unmet need for an appraisal of the quality of RWE in AIT, the European Academy of Allergy and Clinical Immunology Methodology Committee recently initiated a systematic review of observational studies of AIT, which will use the RELEVANT tool and the Grading of Recommendations Assessment, Development and Evaluation approach (GRADE) to rate the quality of the evidence base as a whole. The next step will be to develop a broadly applicable, pragmatic "realworld" database using systematic data collection. Based on the current RWE base, and perspectives and recommendations of authorities and scientific societies, a hierarchy of RWE in AIT is proposed, which places pragmatic trials and registry data at the positions of highest level of evidence. There is a need to establish more AIT registries that collect data in a cohesive way, using standardised protocols. This will provide an essential source of real-world data that can be easily shared, promoting evidence-based research and quality improvement in study design and clinical decision-making.
\end{abstract}

\section{Hosted file}

PAOLETTI complete manuscript with figures and tables.pdf available at https://authorea.com/ users/336456/articles/504275-allergen-immunotherapy-the-growing-role-of-observationaland-randomised-trial-real-world-evidence 